\title{
Insights into the long-to-intermediate-term pre-shock accelerating moment release (AMR) from the March 11, 2011, off the Pacific coast of Tohoku, Japan, $M 9$ earthquake
}

\author{
Changsheng Jiang and Zhongliang Wu \\ Institute of Geophysics, China Earthquake Administration, No. 5 Minzu Daxue Nan Rd., Haidian District, Beijing 100081, China
}

(Received April 7, 2011; Revised March 11, 2012; Accepted March 13, 2012; Online published September 18, 2012)

\begin{abstract}
Great earthquakes with extending rupture areas, such as the March 11, 2011, off the Pacific coast of Tohoku, Japan, $M 9$ earthquake, provide opportunities to inspect some details of the pre-shock moment release with the reference of the earthquake preparation and rupture processes. To this end, we investigated the cumulative seismic moment tensor for different segments of the earthquake fault. For the 3 decades time scale, pre-shock accelerating moment release (AMR) can be observed, with potential correlation with the segmentation of earthquake fault.

Key words: Accelerating moment release, Tohoku M9 earthquake, fault segmentation.
\end{abstract}

\section{Introduction}

In the assessment of long-to-intermediate-term timedependent seismic hazards, one of the candidate models is the pre-shock accelerating moment release (AMR) model (Bufe et al., 1994; Brehm and Braile, 1998; Bowman and King, 2001) which was once related to the criticalpoint-like behavior of earthquake preparation (Sornette and Sammis, 1995; Bowman et al., 1998; Jaumé and Sykes, 1999; Rundle et al., 2000). Regarding this model, present results are somewhat controversial (Robinson, 2000; Chen, 2003; Wang et al., 2004; Robinson et al., 2005; Jiang and Wu, 2006a; Mignan et al., 2006; Papazachos et al., 2007; Hardebeck et al., 2008; Mignan and Giovambattista, 2008; Mignan, 2011). The main discrepancy is whether 'precursory' AMR exists before the 'target' earthquakes, and the discussion has been, to a large extent, influenced by two ambiguities in the AMR analysis. The first ambiguity is that it is the earthquake magnitude (rather than the seismic moment tensor) that is used in the calculation of the 'moment' release-theoretically this is not exact, since the simple use of magnitude is equivalent to summing up all the scalar seismic moments in the Kostrov (1974) method, which is intrinsically not physical. The second ambiguity is that previous studies tended to investigate the seismicity within a large area surrounding a 'target earthquake'. In this case, the AMR behavior reflects a coarse or averaged property of the earthquake preparation process. Considering the complexity of earthquake preparation-for instance, the segmentation of earthquake faults - this average misses some important information of stress variation, if the assumption holds that AMR is related to the pre-shock stress evolution. To a large extent, these two ambiguities

Copyright (C) The Society of Geomagnetism and Earth, Planetary and Space Sciences (SGEPSS); The Seismological Society of Japan; The Volcanological Society of Japan; The Geodetic Society of Japan; The Japanese Society for Planetary Sciences; TERRAPUB.

doi:10.5047/eps.2012.03.002 result from the limitation of earthquake data in use. For an earthquake less than magnitude 7 , the sample of small earthquakes just within the fault zone of the 'target' earthquake, for checking the pre-shock AMR, is small; the seismic moment tensor dataset is even smaller, preventing a detailed and physical investigation of the pre-shock stressrelated 'moment' release. Great earthquakes, characterized by their extending rupture areas, provide unique opportunities to overcome the above-mentioned two shortcomings (Jiang and Wu, 2005, 2006b, 2010; Mignan et al., 2006). In the present investigation related to the March 11, 2011, off the Pacific coast of Tohoku, Japan, $M 9$ earthquake (Lay and Kanamori, 2011; http://www.eqh.dpri.kyotou.ac.jp/src/etc/tohoku/), the seismic moment tensor data (http://www.globalcmt.org/) seems to provide some insights into the pre-shock AMR related to the earthquake preparation and rupture processes.

\section{Data and Background Information}

We used the Global Centroid Moment Tensor (http://www.globalcmt.org/) catalogue for the analysis, while, in principle, the analysis is also valid for other moment tensor datasets. The Gutenberg-Richter frequencymagnitude relation, together with other analyses, gives that the completeness of this catalogue for the Japan islands region is above $M_{\mathrm{w}} 5.2$, satisfying the requirement of AMR analysis for the cutoff magnitude. As in the case of the RELM test (Field, 2007), we have considered both cases with and without de-clustering, and with and without eliminating the 'interfering events'.

Because the earthquakes under consideration are all above $M_{\mathrm{w}} 5.2$, we used the Gardner-Knopoff formula (Gardner and Knopoff, 1974) to identify the aftershocks, which is a simple de-clustering scheme, although less sophisticated than the ETAS model. In the AMR investigation, an 'interfering event' is one located near the 'target event', with a magnitude difference from the 'target event' 
less than 1.0 (e.g., Brehm and Braile, 1998), causing an abrupt change of the AMR curve, which is considered as an artifact in need of elimination. Focusing on the near source areas, we eliminated the 'interfering events' by considering only earthquakes with magnitudes ranging from 5.2 to 6.9 , which is a more stringent elimination to make the cumulative moment release curve smoother. This also eliminates those foreshocks (such as the March 9, 2011, $M 7.5$, foreshock) which are above magnitude 6.9, so that the long-term AMR can be investigated without the influence of short-term, or immediate, foreshocks. Generally, these foreshocks would have a significant effect on the moment release curve if they were not eliminated in the analysis, but what is being focused on is the long-to-intermediateterm cumulative (and nearly gradual) moment release. For reasons of brevity, we present only the case with the elimination of the 'interfering events'. The de-clustering of seismic events showed little effect on the qualitative behavior of the cumulative moment release with regard to accelerating or decelerating.

Traditionally, in the analysis of seismicity (e.g., KeilisBorok and Rotwain, 1990), accounting for the quality of depth determination in the earthquake catalogue, the analysis simply considers all the 'shallow' earthquakes with depths ranging from 0 to $70 \mathrm{~km}$. In this investigation, we followed the same convention. However, since the rupture of this great earthquake is large, with a low dip angle, the depth distribution can be 'transformed' into a horizontal distribution. Figure 1 shows the rupture of the March 11, 2011, M 9 mainshock (after Lay and Kanamori, 2011), together with the key areas subject to AMR analysis. It can be seen that the availability of enough moment tensor data is, to a large extent, due to the size of the earthquake rupture.

After the March 11, 2011, Tohoku earthquake, several studies have been published relating to the seismicity before, and after, the mainshock. Ishikawa (see, http://www.ab.cyberhome.ne.jp//catfish/event/2011ejapan.html) provided a quick analysis of regional and global seismicity before, and after, the Tohoku earthquake. Seismicity was systematically outlined by Hirose et al. (2011). Katsumata (2011) discussed the long-term seismic quiescence which started some 20 years before the earthquake. The Tohoku earthquake also raises an unprecedented question regarding the along-dip segmentation versus the alongstrike segmentation (Yomogida et al., 2011). According to the study of the rupture process (see, Lay and Kanamori, 2011, for a concise review), the earthquake rupture starts from the deeper part radiating high-frequency-predominant seismic waves, and continues by the shallower part radiating low-frequency-predominant seismic waves and generating tsunami-generic displacements. This along-dip segmentation characteristic is different from previous great earthquakes, such as the Sumatra-Andaman earthquake in 2004. In this study, therefore, we have investigated the cumulative moment release within several specific regions, as shown in Fig. 1. With regard to methodology, this idea is somehow similar to the Reverse Tracing of Precursors (RTP, see: Keilis-Borok et al., 2004), with the difference that the RTP considers long-term anomalies within the areas of shortterm precursory anomalies, but the present approach inves-

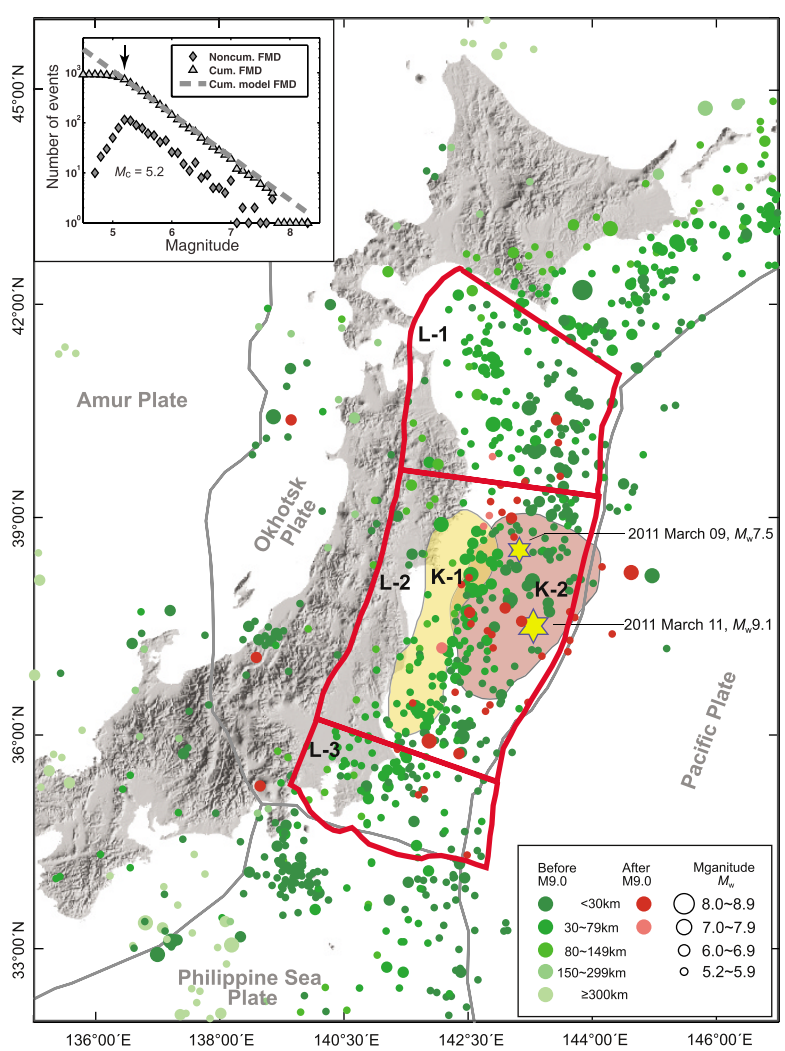

Fig. 1. The seismic rupture of the March 11, 2011, M9 earthquake ( $\left.M_{\mathrm{W}} 9.0 / \mathrm{NEIC}, M_{\mathrm{w}} 9.1 / \mathrm{GCMT}\right)$, and the distribution of earthquakes subject to analysis. Hexagons give the centroids of the mainshock and its immediate foreshock, respectively. Plate boundaries, as shown by dark gray lines, are from Bird (2003). In the figure, 5 areas for the AMR analysis, L-1, L-2, L-3, K-1 and K2, are marked (for the seismological significance of these areas, refer to Lay and Kanamori, 2011). Note that the boundaries of these areas consider both tectonics and seismic clustering. Top left subplot is the frequency-magnitude distribution of the earthquakes under consideration, giving the completeness magnitude.

tigates AMR within the specific areas related to the earthquake preparation and rupture processes.

\section{Characterizing the Long-to-intermediate-term Moment Release}

Whether the seismicity exhibits an accelerating behavior is described by fitting the cumulative moment release curve with a linear function and a power-law function, respectively. Eliminating the 'interfering events', this curve is relatively 'smooth' and reflects the long-to-intermediateterm deformation. Similar to previous works such as Bufe and Varnes (1993) and Bowman et al. (1998), which are especially for analyzing the cumulative Benioff strain, the power-law function for a moment tensor element $M_{i j}$ can be expressed by

$$
\sum M_{i j}=A+B\left(t_{0}-t\right)^{m}
$$

In the equation, the left-hand side is the summation of the moment tensor element under consideration, which is similar to the formulation of Kostrov (1974) if divided by the area under consideration. In contrast, traditional AMR analysis corresponds to the summation of the absolute values (or their square root) of the moment tensor element, being less physical. On the right-hand side of the equation, 

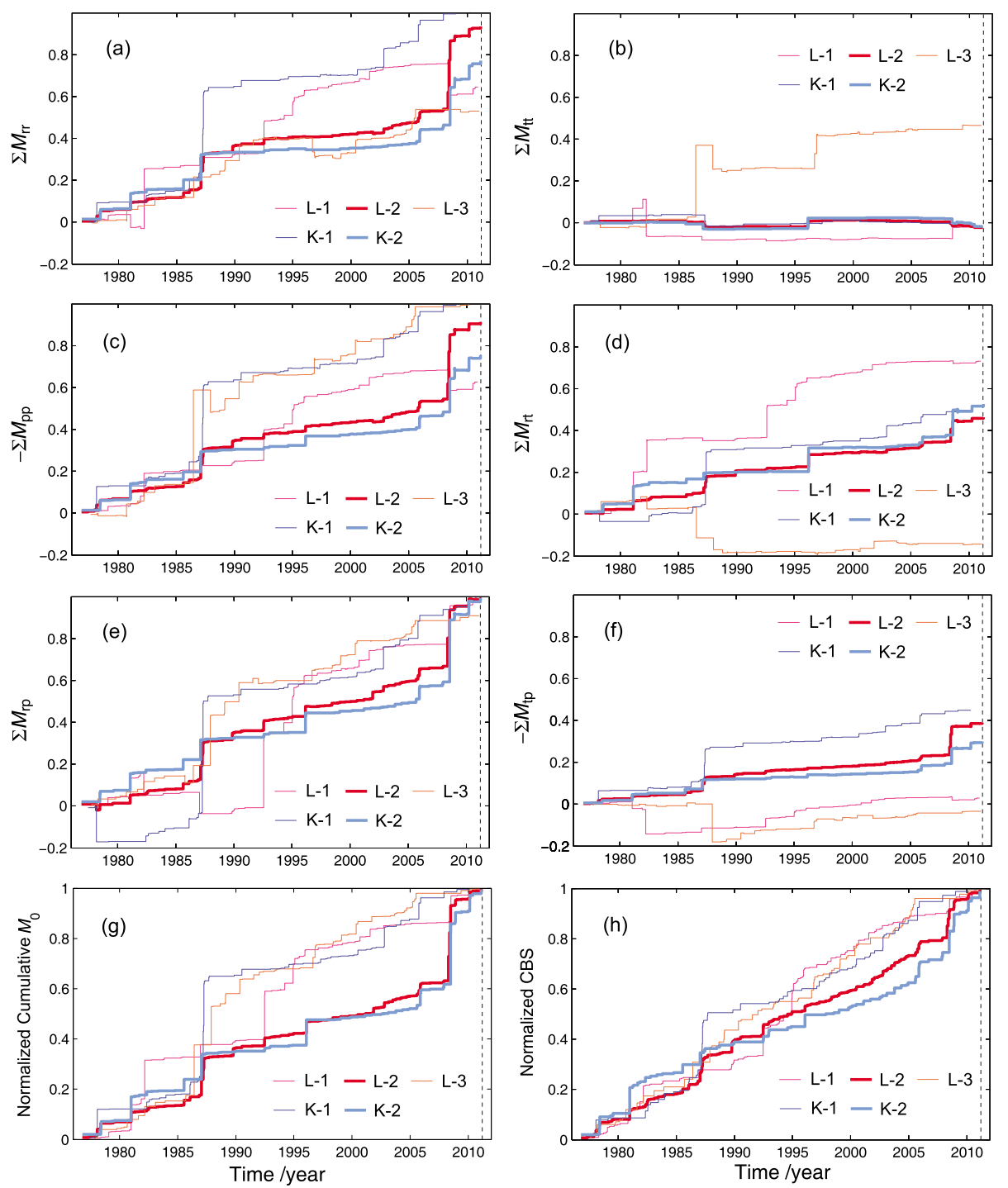

Fig. 2. Cumulative seismic moment tensor elements (a)-(f), cumulative scalar seismic moment (g), and cumulative Benioff strain (h, as represented by the cumulative square root of the scalar seismic moment). Different line types correspond to the 5 areas shown in Fig. 1. In Figs. 2(a)-(f), for the 5 areas, respectively, the moment tensor elements are normalized by the maximum cumulative element, so that the accelerating/decelerating trend may be clearly visible and compared with each other. The vertical dashed line shows the time of the Tohoku earthquake. Note that the abrupt variations in the cumulative moment tensor element curves, such as that of L-2 in the period from 2005 to 2010, are not caused by a single event but a series of events accumulating.

$A$ and $B$ are constants, $t_{0}$ is the 'failure time' of the 'target' earthquake, and $m$ the power-law exponent. By definition, $m$ less than 1 indicates the accelerating-like case; $m$ larger than 1 indicates the decelerating-like case; and $m$ near unity indicates the linear case. In this investigation, to focus on whether AMR exists by the exponent $m$, the failure time $t_{0}$ was fixed to be the origin time of the March 11, 2011, M9 earthquake, so that the fitting could be stabilized by reducing one free parameter, $t_{0}$. In fitting Eq. (1), depending on the datasets, the uncertainty of the $m$ value, $\Delta m$, can also be obtained. Another factor to be considered is to avoid the problem of over-fitting. To this end, we use the criteria that, comparing to a linear fit, the BIC gain of the power-law fit has to be positive-otherwise the moment release is not statistically-significant accelerating/decelerating. The BIC gain for AMR is a development of the 'curvature parameter' $C$ (Bowman et al., 1998) defined by the quotient of the RMS residual for the power-law fit with that for the linear fit, being prescribed (somewhat arbitrarily) that the case with $C$ smaller than 0.70 is 'significantly' accelerating/decelerating. Using the BIC gain to the AMR studies (Jiang and Wu, 2010) taking the simplified form of BIC (Seher and Main, 2004), represented by $[-(N / 2) \ln (\mathrm{RMS})-(p / 2) \ln (N / 2 \pi)]$ in which $N$ is the number of data samples and $p$ the degrees-of-freedom of the model, a 'reasonable' AMR fitting is defined more 'objectively' by the criteria $\triangle \mathrm{BIC}>0$ in comparison with a linear fit. That is, over-fitting due to the use of too many free parameters (or few data samples) will be penalized by the BIC function which includes both $p$ and $N$.

\section{Results and Discussion}

In the present work, we used a two-level spatial scope, as shown in Fig. 1: the areas L-1, L-2, and L-3, with reference to the GPS-measured locking status (Loveless and Meade, 2010; Lay and Kanamori, 2011); and the areas K-1 and K-2, 
(a)
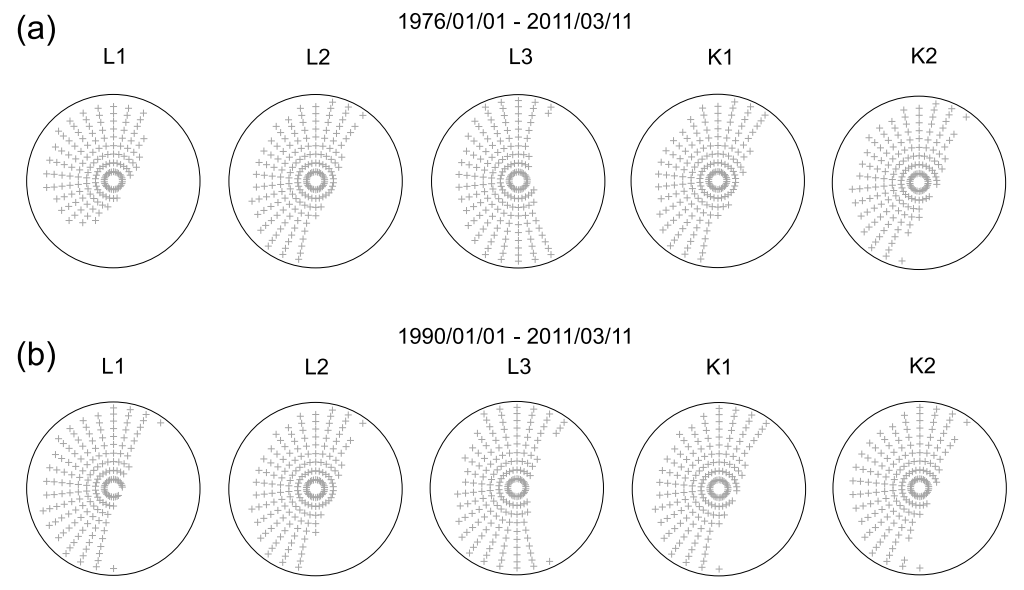

1990/01/01-2011/03/11

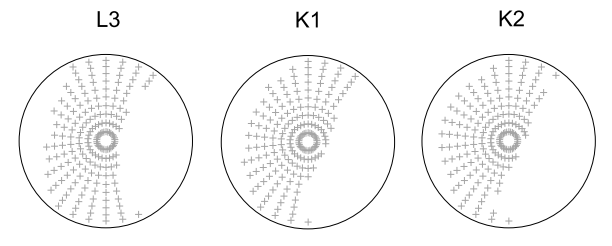

(c)

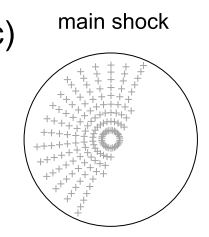

Fig. 3. Equal-area projection of the cumulative moment tensors before the March 11, 2011, $M 9$ mainshock, for the 5 areas shown in Fig. 1. (a) Since 1976/01/01; (b) Since 1990/01/01; (c) Focal mechanism of the March 11, 2011, M 9 mainshock (from GCMT).

with reference to the earthquake rupture process (Koper et al., 2011; Lay and Kanamori, 2011). L-2 is the area accommodating the source of the great earthquake; and the great earthquake was started by K-1 and followed by K-2 (Lay and Kanamori, 2011). Similar to Yomogida et al. (2011) but with a different spatial scale, these areas can be naturally divided by the clustering of seismicity. Limitation of samples did not provide a statistically-reliable AMR result with respect to the along-strike segmentation of Yomogida et al. (2011) and is not presented here due to limitations of space. Figure 2 shows the cumulative seismic moment tensor elements (a-f), the cumulative scalar seismic moment $(\mathrm{g})$, and the cumulative Benioff strain (h, as represented by the cumulative square root of the scalar seismic moment), respectively, for the 5 areas shown in Fig. 1. In Figs. 2(a)-(f), for the 5 areas, respectively, the moment tensor elements are normalized by the maximum element, so that the accelerating/decelerating trend may be clearly visible and compared with each other. One of the main differences between the moment tensor summation (Kostrov, 1974) and the scalar moment summation is that the latter always provides a monotonically increasing curve, but the former does not. From the variation of different moment tensor elements it may be seen that the cumulative moment tensor is controlled by a few predominant elements, indicating the directivity of the focal mechanisms. Figure 3 further shows that these cumulative moment tensors are, to a differing extent, similar to that of the March 11, 2011, mainshock. Also note that the abrupt variations in the cumulative moment tensor element curves, such as that of L-2 in the period from 2005 to 2010 , are not caused by a single event but a series of events accumulating.

Parameters of the fitting of the cumulative scalar seismic moment curve $(\mathrm{g})$ and the curve of the cumulative square root of the scalar moment $(\mathrm{h}$, corresponding to the cumu-
Table 1. Parameters of the fitting*.

\begin{tabular}{ccccccccc}
\hline & \multicolumn{3}{c}{ Scalar moment curve } & \multicolumn{3}{c}{ Benioff strain curve } \\
\cline { 2 - 9 } Area & $m$ & $\Delta m$ & $m$ & $\Delta m$ & $m$ & $\Delta m$ & $m$ & $\Delta m$ \\
\hline L-1 & 1.54 & 0.16 & 3.15 & 0.46 & 1.08 & 0.10 & 2.15 & 0.20 \\
L-2 & $\mathbf{0 . 5 4}$ & $\mathbf{0 . 0 7}$ & $\mathbf{0 . 3 7}$ & $\mathbf{0 . 0 7}$ & $\mathbf{0 . 8 5}$ & $\mathbf{0 . 0 4}$ & $\mathbf{0 . 6 0}$ & $\mathbf{0 . 0 4}$ \\
L-3 & 1.83 & 0.20 & 1.25 & 0.16 & 1.17 & 0.06 & 1.15 & 0.13 \\
K-1 & 1.72 & 0.44 & 0.46 & 0.26 & 1.15 & 0.19 & 0.74 & 0.18 \\
K-2 & $\mathbf{0 . 4 6}$ & $\mathbf{0 . 0 8}$ & $\mathbf{0 . 4 3}$ & $\mathbf{0 . 1 1}$ & $\mathbf{0 . 5 9}$ & $\mathbf{0 . 0 7}$ & 0.50 & $\mathbf{0 . 0 7}$ \\
\hline
\end{tabular}

* Note: For all the cases, BIC gain is positive. AMR-like cases are marked with bold.

lative Benioff strain traditionally used in AMR studies), respectively, are shown in Table 1, to check the statistical significance of the accelerating/decelerating behavior. In Table 1, two time scales are considered: the time period since 1976 (the starting time of the GCMT catalogue), and the time since 1990, which correspond to 3.5 decades and 2 decades time scale, respectively. From the table it may be seen that an apparent AMR exists for the L-2 and K-2 areas, as shown by the $m$ exponent. From the Benioff strain curve, it is difficult to judge whether L-1, L-3, and K-1 are decelerating or linearly increasing, although the cumulative scalar moment curve indicates a decelerating-like trend. K1 seems to exhibit an accelerating trend at the 2 decades time scale. But for the cumulative Benioff strain curve, $(m+\Delta m)$ almost becomes 1 , therefore the seemingly accelerating trend is suspicious. On the other hand, from either the Benioff strain or the scalar moment, L-2 and K-2 are significantly accelerating, within the 3.5 decades and 2 decades time scale. From Fig. 3 it may also be seen that the cumulative moment tensors for $\mathrm{L}-2, \mathrm{~K}-1$, and $\mathrm{K}-2$ are more similar to that of the March 11 mainshock. This also provides an opportunity to judge the 'direction of the acceleration', which is beyond the scope of this short letter. 


\section{Concluding Remarks}

The March 11, 2011, off the Pacific coast of Tohoku Japan, $M 9$ earthquake casts a new light on the study of the long-to-intermediate-term moment release problem. Due to its retrospective nature, this study has a very limited significance for 'real forward' time-dependent seismic hazard assessment. Remarkably, from the cumulative moment curves the 'starting time' of the accelerating stage would be difficult to judge if there were really an AMR process. Due to this reason, fitting of the 'failure time' is not within the scope of this work, which simply investigates whether or not AMR exists - a simpler but still tough problem. Seen from the moment release curve at the decades time scale, it is also difficult to differentiate an accelerating process and a quiescence-and-recovery process. However, results from the analysis may provide some heuristic clues to the understanding of the relation between the observed AMR and the process of earthquake preparation. Note that the L-2 area, showing AMR behavior, is that accommodating the great earthquake, which may not be surprising. But note that K-2 is the area that produces the latter initiated, tsunami generic slip, it seems that although the K-2 rupture is somehow a follow-up of the rupture in K-1, the preparation of the rupture of this area has been at least 3 decades, as indicated by the AMR behavior.

Acknowledgments. We thank the anonymous referees as well as the editor for constructive suggestions in revising the manuscript. The study is supported by the international science and technology cooperation project (Grant No. 2012DFG20510), from the Ministry of Science and Technology of China.

\section{References}

Bird, P., An updated digital model of plate boundaries, Geochem. Geophys. Geosyst., 4, 1027, doi:10.1029/2001GC000252, 2003.

Bowman, D. D. and G. C. P. King, Accelerating seismicity and stress accumulation before large earthquakes, Geophys. Res. Lett., 28, 40394042, 2001.

Bowman, D. D., G. Ouillon, C. G. Sammis, A. Sornette, and D. Sornette, An observational test of the critical earthquake concept, J. Geophys. Res., 103, 24359-24372, 1998.

Brehm, D. J. and L. W. Braile, Intermediate-term earthquake prediction using precursory events in the New Madrid seismic zone, Bull. Seismol. Soc. Am., 88, 564-580, 1998.

Bufe, C. G. and D. J. Varnes, Predictive modeling of the seismic cycle of the Greater San Francisco Bay Region, J. Geophys. Res., 98, 98719883, 1993.

Bufe, C. G., S. P. Nishenko, and D. J. Varnes, Seismicity trends and potential for large earthquake in the Alaska-Aleutian region, Pure Appl. Geophys., 142, 83-99, 1994.

Chen, C. C., Accelerating seismicity of moderate-size earthquakes before the 1999 Chi-Chi, Taiwan, earthquake: Testing time-prediction of the self-organizing spinodal model of earthquakes, Geophys. J. Int., 155, F1-F5, doi:10.1046/j.1365-246X.2003.02071.x, 2003.

Field, E. D. (eds.), Special Issue-Regional Earthquake Likelihood Models, Seismol. Res. Lett., 78, 1-140, 2007.

Gardner, J. K. and L. Knopoff, Is the sequence of earthquakes in Southern California, with aftershocks removed, Poissonian?, Bull. Seismol. Soc. Am., 64, 1363-1367, 1974.

Hardebeck, J. L., K. R. Felzer, and A. J. Michael, Improved tests reveal that the accelerating moment release hypothesis is statistically insignificant, J. Geophys. Res., 113, B08310, doi:10.1029/2007JB005410, 2008.

Hirose, F., K. Miyaoka, N. Hayashimoto, T. Yamazaki, and M. Nakamura, Outline of the 2011 off the Pacific coast of Tohoku Earthquake $\left(M_{\mathrm{w}}\right.$ 9.0)-Seismicity: foreshocks, mainshock, aftershocks, and induced activity-, Earth Planets Space, 63, 513-518, 2011.

Jaumé, S. C. and L. R. Sykes, Evolving towards a critical point: A review of accelerating seismic moment/energy release prior to large and great earthquake, Pure Appl. Geophys., 155, 279-306, 1999.

Jiang, C. S. and Z. L. Wu, Test of the preshock accelerating moment release (AMR) in the case of the 26 December 2004 Indonesia $M_{\mathrm{w}} 9.0$ earthquake, Bull. Seismol. Soc. Am., 95, 2026-2035, doi:10.1785/0120050018, 2005.

Jiang, C. S. and Z. L. Wu, Benioff strain release before earthquakes in China: Accelerating or not?, Pure Appl. Geophys., 163, 1965-1976, $2006 a$.

Jiang, C. S. and Z. L. Wu, Pre-shock seismic moment release in different segments of an earthquake fault: The case of the December 26, 2004 Indonesia $M_{w} 9.0$ earthquake, $A d v$. Geosci., 1, 17-25, 2006b.

Jiang, C. S. and Z. L. Wu, Seismic moment release before the May 12, 2008, Wenchuan earthquake in Sichuan of southwest China, Concurrency Comput.: Pract. Exper., 22, 1784-1795, doi:10.1002/cpe.1522, 2010.

Katsumata, K., A long-term seismic quiescence started 23 years before the 2011 off the Pacific coast of Tohoku Earthquake $(M=9.0)$, Earth Planets Space, 63, 709-712, 2011.

Keilis-Borok, V. I. and I. M. Rotwain, Diagnosis of time of increased probability of strong earthquakes in different regions of the world, Phys. Earth Planet. Inter., 61, 57-72, 1990.

Keilis-Borok, V., P. Shebalin, A. Gabrielov, and D. Turcotte, Reverse tracing of short-term earthquake precursors, Phys. Earth Planet. Inter., 145, 75-85, 2004.

Koper, K. D., A. R. Hutko, T. Lay, C. J. Ammon, and H. Kanamori, Frequency-dependent rupture process of the $2011 M_{\mathrm{w}} 9.0$ Tohoku Earthquake: Comparison of short-period $P$ wave backprojection images and broadband seismic rupture models, Earth Planets Space, 63, 599602, 2011.

Kostrov, B. V., Seismic moment and energy of earthquakes, and seismic flow of rock, Izv. Acad. Sci. USSR Phys. Solid Earth, 1, 23-40, 1974.

Lay, T. and H. Kanamori, Insights from the great 2011 Japan earthquake, Phys. Today, December 2011, 33-39, 2011.

Loveless, J. P. and B. J. Meade, Geodetic imaging of plate motions, slip rates, and partitioning of deformation in Japan, J. Geophys. Res., 115, B02410, doi:10.1029/2008JB006248, 2010.

Mignan, A., Retrospective on the Accelerating Seismic Release (ASR) hypothesis: Controversy and new horizons, Tectonophysics, 505, 1-16, doi:10.1016/j.tecto.2011.03.010, 2011.

Mignan, A. and R. D. Giovambattista, Relationship between accelerating seismicity and quiescence, two precursors to large earthquakes, Geophys. Res. Lett., 35, L15306, doi:10.1029/2008GL035024, 2008.

Mignan, A., G. King, D. Bowman, R. Lacassin, and R. Dmowska, Seismic activity in the Sumatra-Java region prior to the December 26, 2004 $\left(M_{w}=9.0-9.3\right)$ and March $28,2005\left(M_{w}=8.7\right)$ earthquakes, Earth Planet. Sci. Lett., 244, 639-654, 2006.

Papazachos, B. C., E. M.Scordilis, D. G. Panagiotopoulos, C. B. Papazachos, and G. F. Karakaisis, Currently active regions of deceleratingaccelerating seismic strain in central Asia, J. Geophys. Res., 112, B10309, doi:10.1029/2006JB004587, 2007.

Robinson, R., A test of the precursory accelerating moment release model on some recent New Zealand earthquakes, Geophys. J. Int., 140, 568576, 2000.

Robinson, R., S. Y. Zhou, S. Johnston, and D. Vere-Jones, Precursory accelerating seismic moment release (AMR) in a synthetic seismicity catalog: A preliminary study, Geophys. Res. Lett., 32, L07309, doi:10.1029/2005GL022576, 2005.

Rundle, J. B., W. Klein, D. L. Turcotte, and B. D. Malamud, Precursory seismic activation and critical-point phenomena, Pure Appl. Geophys., 157, 2165-2182, 2000.

Seher, T. and I. G. Main, A statistical evaluation of a 'stress-forecast' earthquake, Geophys. J. Int., 157, 187-193, 2004.

Sornette, D. and C. G. Sammis, Critical exponents from renormalization group theory of earthquakes: Implications for earthquake prediction, $J$. Phys. I., 5, 607-619, 1995.

Wang, Y. C., C. Yin, P. Mora, X. C. Yin, and K. Y. Peng, Spatio-temporal scanning and statistical test of the accelerating moment release (AMR) model using Australian earthquake data, Pure Appl. Geophys., 161, 2281-2293, 2004.

Yomogida, K., K. Yoshizawa, J. Koyama, and M. Tsuzuki, Along-dip segmentation of the 2011 off the Pacific coast of Tohoku Earthquake and comparison with other megathrust earthquakes, Earth Planets Space, 63, 697-701, 2011.

C. Jiang and Z.Wu (e-mail: wuzl@cea-igp.ac.cn) 